\title{
TRANSIENT RESPONSE OF A DISTRIBUTED $R L C$ INTERCONNECT BASED ON DIRECT POLE EXTRACTION
}

\author{
GUOQING CHEN* and EBY G. FRIEDMAN \\ Department of Electrical and Computer Engineering, \\ University of Rochester, Rochester, NY 1462\%, USA \\ *guoqing.chen@intel.com
}

Revised 28 June 2009

\begin{abstract}
With higher operating frequencies, transmission lines are required to model global onchip interconnects. In this paper, an accurate and efficient solution for the transient response at the far end of a transmission line based on a direct pole extraction of the system is proposed. Closed form expressions of the poles are developed for two special interconnect systems: an $R C$ interconnect and an $R L C$ interconnect with zero driver resistance. By performing a system conversion, the poles of an interconnect system with general circuit parameters are solved. The Newton-Raphson method is used to further improve the accuracy of the poles. Based on these poles, closed form expressions for the step and ramp response are determined. Higher accuracy can be obtained with additional pairs of poles. The computational complexity of the model is proportional to the number of pole pairs. With two pairs of poles, the average error of the $50 \%$ delay is $1 \%$ as compared with Spectre simulations. With ten pairs of poles, the average error of the $10 \%$-to- $90 \%$ rise time and the overshoots is $2 \%$ and $1.9 \%$, respectively. Frequency dependent effects are also successfully included in the proposed method and excellent match is observed between the proposed model and Spectre simulations.
\end{abstract}

Keywords: Interconnect; modeling; transient response; pole.

\section{Introduction}

With increasing on-chip signal frequencies, the effect of interconnect inductance has become more significant, particularly in global interconnects. Furthermore, the clock period is continuously decreasing. The timing characteristics of on-chip signals therefore need to be determined and controlled more precisely. Accurate interconnect models, such as $R L C$ transmission lines, and efficient solutions to analyze on-chip interconnects are required in the integrated circuit design process. ${ }^{1,2}$

Sakurai presented an accurate closed-form solution for distributed $R C$ interconnect based on a single pole approximation in Refs. 3-4. By truncating the transfer function, multi-pole models have been proposed in the last decade to capture the

\footnotetext{
${ }^{*}$ Guoqing Chen is currently with Intel Corporation, Folsom, CA.
} 
effect of inductance; for example, two poles in Ref. 5 and four poles in Ref. 6 . No closed-form solution, however, is provided for the four-pole method. In Ref. 7, the solution for an open-ended interconnect with a step input signal is rigorously developed. This solution is however highly complicated and not suitable for an exploratory design process. In Ref. 8, a traveling wave analysis (TWA) model has been presented, where the key points of the waveform are determined with a threepole model, and linear or $R C$ approximations are used to connect those key points to construct the waveform. This method is improved in Ref. 9, where the key points and slopes are more accurately determined with the model described in Ref. 7, and straight lines are used to construct the signal waveforms in different time regions. In both of these papers, the output response is divided into a number of time regions where the waveform expressions for each of the regions are different, making the models less compact. Furthermore, none of these aforementioned papers consider frequency dependent effects. With higher on-chip frequencies, frequency dependent effects in wider interconnect can no longer be ignored. In Ref. 10, a Fourier analysis based interconnect model is proposed, where the far end response is approximated by the first several harmonics. Frequency dependent effects can be included in this model; however, the model is only suitable for periodic signals. In all of these papers, a uniform wire impedance is assumed. In advanced global interconnect structures, such as a network-on-chip, ${ }^{11}$ interconnects are regularly designed on the topmost layers. At early design stages, the layout describing the orthogonal layers is not available. Treating the orthogonal layer as a ground plane is a reasonable assumption for capacitance extraction. ${ }^{12}$ To control chemical mechanical polishing (CMP) induced process variations, dummy fillings are often inserted on-chip to achieve a uniform pattern density, making a uniform impedance assumption more accurate.

Moment matching method ${ }^{13}$ and Krylov-subspace-based model order reduction techniques, such as Pade via Lanczos (PVL), ${ }^{14}$ Arnoldi algorithm, ${ }^{15}$ and passive reduced order interconnect macromodeling algorithm (PRIMA), ${ }^{16}$ have been widely used in solving complex interconnect structures. With transmission line effect becoming more significant, additional $R L C$ segments need to be used to model the distributed interconnect behavior. The computational efficiency of these method will be reduced, making them unsuitable for single interconnect analysis in early design stages.

In Ref. 17, a new method for computing the far end response of a transmission line is proposed. The model is based on a direct pole extraction of the exact transfer function of a transmission line, rather than approximating the poles by truncating the transfer function ${ }^{5,6}$ or matching moments. ${ }^{13}$ Closed-form waveform expressions are developed, permitting flexible trade-offs between accuracy and efficiency. This model is extended in this paper by including frequency dependent effects. The poles of $R C$ interconnect are also determined analytically. The rest of the paper is organized as follows. In Sec. 2, the exact poles of two special case interconnect systems are determined. Based on these poles, the step and ramp responses are developed. In Sec. 3, an interconnect system with general circuit parameters is 
solved. The Newton-Raphson method is used to determine the exact poles of the system. Frequency dependent effects are successfully included in Sec. 4. Finally, some conclusions are offered in Sec. 5 .

\section{Special Cases of a Single Interconnect System}

For a distributed $R L C$ interconnect driven by a voltage source with a driver resistance $R_{d}$ and loaded with a lumped capacitance $C_{L}$, as shown in Fig. 1, the transfer function is 5,10

$$
H(s)=\frac{1}{\left(1+R_{d} C_{L} s\right) \cosh (\theta)+\left(R_{d} / Z_{c}+Z_{c} C_{L} s\right) \sinh (\theta)},
$$

where $\theta=\sqrt{(R+L s) C s}$ and $Z_{c}=\sqrt{(R+L s) / C s}=\theta / C s . R, L$, and $C$ are, respectively, the resistance, inductance, and capacitance of the interconnect. The poles of Eq. (1) are difficult to solve directly except for two special cases: an $R C$ interconnect and an $R L C$ interconnect with a zero driver resistance. In Sec. 2.1, the poles of an $R C$ interconnect system are solved. In Sec. 2.2, the poles of an $R L C$ interconnect with a zero driver resistance are solved. Step and ramp responses are developed in Sec. 2.3.

\subsection{RC interconnect}

For $R C$ interconnect, $L=0$. The transfer function Eq. (1) can be rewritten as

$$
H(s)=\frac{1}{\left(1+A \theta^{2}\right) \cosh (\theta)+B \theta \sinh (\theta)},
$$

where $A=R_{T} C_{T}, B=R_{T}+C_{T}, R_{T}=R_{d} / R, C_{T}=C_{L} / C$, and $\theta=\sqrt{R C s}$. Let $F(s)=1 / H(s)$. The poles of $H(s)$ are zeros of $F(s)$ and satisfy $F(s)=0$. Observe that $\theta$ needs to be an imaginary number to make $F(s)$ zero. Assume $\theta=j x$, where $x$ is a real number. Expression $F(s)=0$ can be transformed to

$$
\left(1-A x^{2}\right) \cos x-B x \sin x=0,
$$

or

$$
\tan x=\frac{1-A x^{2}}{B x} .
$$

The roots of Eq. (4) are the crossing points of the functions of $y=\tan x$ and $y=\left(1-A x^{2}\right) /(B x)$, as shown in Fig. 2 .

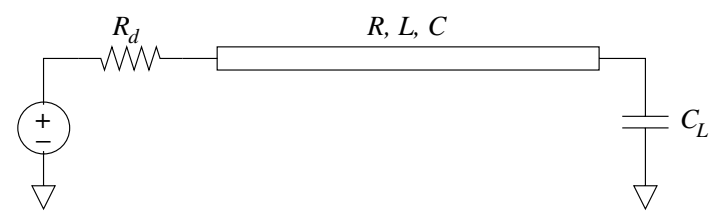

Fig. 1. Distributed interconnect with a lumped capacitive load and driver resistance. 


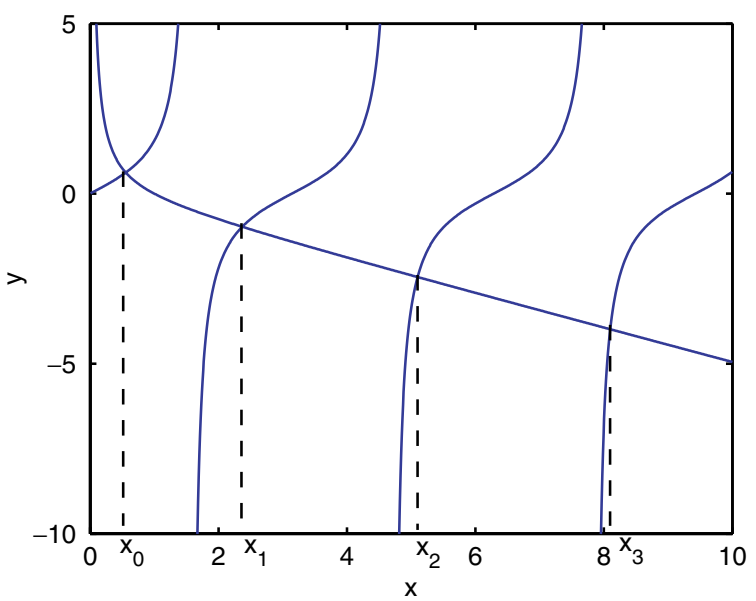

Fig. 2. Graphic view of the roots of (4), $R_{T}=C_{T}=1$.

Applying Taylor series expansions of $\cos x \approx 1-x^{2} / 2+x^{4} / 24$ and $\sin x \approx$ $x-x^{3} / 6$ to Eq. (3), and ignoring those terms with an order higher than $x^{4}, x^{2}$ can be obtained as

$$
x_{0}^{2}=\frac{\frac{1}{2}+A+B-\sqrt{(A+B)^{2}-A+\frac{1}{3} B+\frac{1}{12}}}{A+\frac{1}{3} B+\frac{1}{12}} .
$$

When $R_{T}=C_{T}=0$, the exact value of $x_{0}^{2}$ is $\pi^{2} / 4$. In order to capture this trend, Eq. (5) is revised to

$$
x_{0}^{2}=\frac{\frac{1}{2}+A+B-\sqrt{(A+B)^{2}-A+\frac{1}{3} B+\frac{1}{11.54}}}{A+\frac{1}{3} B+\frac{1}{12}} .
$$

Note that if the terms higher than $x^{2}$ are omitted after applying Taylor expansions, the solution simplifies to

$$
x_{0}^{2}=\frac{1}{0.5+A+B}=\frac{1}{0.5+R_{T}+C_{T}+R_{T} C_{T}},
$$

which is similar to the solution provided in Ref. 4 .

Since the Taylor series approximations used above are expanded around zero, the solution shown in Eq. (6) corresponds to the root $x_{0}$ which is most close to zero, as shown in Fig. 2. In order to obtain other high order solutions, Taylor series approximations expanded at $n \pi(n=1,2, \ldots)$ are used. Since the negative roots of Eq. (3) have the same absolute value as the positive roots, only positive roots are considered in this paper. In determining the timing response of an interconnect, lower order poles are the most important; the accuracy of the higher order poles can be lower. In order to produce a closed-form solution for enhanced computational efficiency, a second order Taylor expansion is used for case $n \geq 1$. Let $\Delta x=x-n \pi$, 
$\cos x \approx(-1)^{n}\left[1-(\Delta x)^{2} / 2\right]$, and $\sin x \approx(-1)^{n} \Delta x$. Substituting these Taylor series approximations into Eq. (3) and ignoring those terms with an order higher than $(\Delta x)^{2}$ results in

$$
\left(A+B-\frac{1}{2} E\right)(\Delta x)^{2}+(2 A+B) n \pi \Delta x+E=0,
$$

where $E=A n^{2} \pi^{2}-1$. Solving Eq. (8) for $x_{n}$ results in

$$
x_{n}=\frac{-(2 A+B) n \pi+\sqrt{(n \pi B)^{2}+4(A+B)+2 E^{2}}}{2(A+B)-E}+n \pi .
$$

The accuracy of Eqs. (6) and (9) is illustrated in Fig. 3 for different values of $R_{T}$ and $C_{T}$. The exact solution is obtained numerically. As shown in Fig. 3, the error of the higher order solutions is larger for greater values of $R_{T}$ and $C_{T}$. In these cases, the effect of the higher order solutions on the timing responses is, however, negligible.

After solving $x_{n}$, the poles of an $R C$ interconnect system can be obtained,

$$
p_{n}=\frac{\theta^{2}}{R C}=\frac{-x_{n}^{2}}{R C}, \quad n=0,1,2, \ldots .
$$

The residue of the corresponding poles is

$$
\begin{aligned}
k_{n} & =\lim _{s \rightarrow p_{n}} \frac{s-p_{n}}{F(s)}=\frac{1}{F^{\prime}\left(p_{n}\right)} \\
& =\frac{2 x_{n} /(R C)}{\left(1+B-A x_{n}^{2}\right) \sin x_{n}+(2 A+B) x_{n} \cos x_{n}},
\end{aligned}
$$

where $F^{\prime}\left(p_{n}\right)$ is the derivative of $F(s)$ at $p_{n}$.

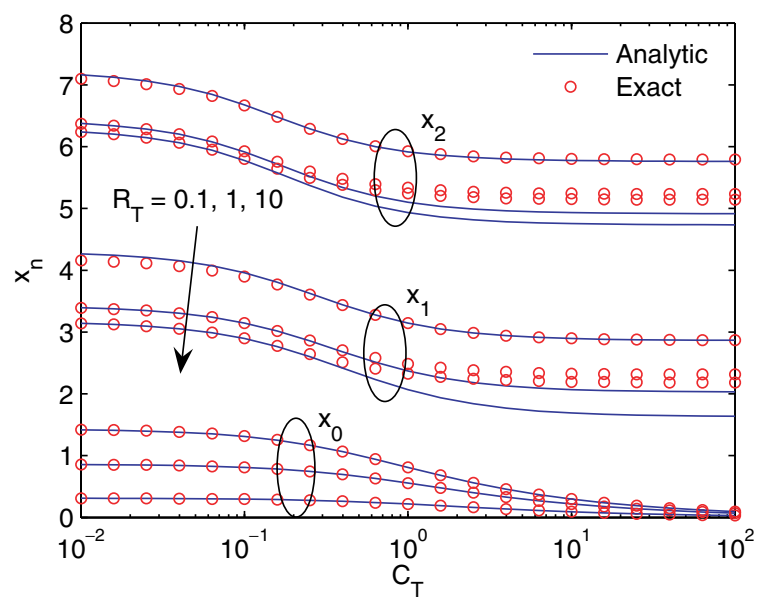

Fig. 3. Analytic solution of Eq. (3) as compared with the exact solution for different values of $R_{T}$ and $C_{T}$. 


\subsection{RLC interconnect with a zero $R_{d}$}

If $R_{d}$ is zero, Eq. (1) simplifies to

$$
H(s)=\frac{1}{\cosh (\theta)+C_{T} \theta \sinh (\theta)} .
$$

Note that $\theta$ also needs to be an imaginary number to make $F(s)$ zero. Similar to the approach for the $R C$ case, assume $\theta=j x$, where $x$ is a real number. The poles of the transfer function should satisfy

$$
\cos x-C_{T} x \sin x=0,
$$

or

$$
x=\frac{\cot x}{C_{T}} .
$$

By applying Taylor series approximations (fourth-order approximation for $n=0$ and second-order approximation for $n \geq 1$ ), $x$ can be solved as

$$
x_{n}= \begin{cases}\sqrt{\frac{\frac{1}{2}+C_{T}-\sqrt{C_{T}^{2}+\frac{1}{3} C_{T}+\frac{1}{12}}}{\frac{1}{3} C_{T}+\frac{1}{12}}}, & n=0, \\ \frac{\left(1+C_{T}\right) n \pi+\sqrt{\left(C_{T} n \pi\right)^{2}+2+4 C_{T}}}{1+2 C_{T}}, & n \geq 1 .\end{cases}
$$

Note that when $C_{T}$ approaches zero, Eq. (13) becomes $\cos x=0$, and the solution $x_{n}$ approaches $(n+1 / 2) \pi$, where $n=0,1,2, \ldots$ In order to capture this trend, Eq. (15) is revised as

$$
x_{n}= \begin{cases}\sqrt{\frac{\frac{1}{2}+C_{T}-\sqrt{C_{T}^{2}+\frac{1}{3} C_{T}+\frac{1}{11.54}}}{\frac{1}{3} C_{T}+\frac{1}{12}}}, & n=0, \\ \frac{\left(1+C_{T}\right) n \pi+\sqrt{\left(C_{T} n \pi\right)^{2}+\frac{\pi^{2}}{4}+4 C_{T}}}{1+2 C_{T}} & n \geq 1 .\end{cases}
$$

The accuracy of Eq. (16) is illustrated in Fig. 4 for different values of $C_{T}$. The exact solution is obtained numerically. As shown in Fig. 4, when $C_{T}$ increases from zero to infinity, $x_{n}$ decreases from $(n+1 / 2) \pi$ to $n \pi$.

The poles of the transfer function can be obtained from the following expression,

$$
L C s^{2}+R C s=\theta^{2}=-x_{n}^{2}, \quad n=0,1,2, \ldots .
$$

Each $x_{n}$ corresponds to a pair of poles,

$$
p_{n, \pm}=\frac{-R C \pm \sqrt{R^{2} C^{2}-4 L C x_{n}^{2}}}{2 L C} .
$$




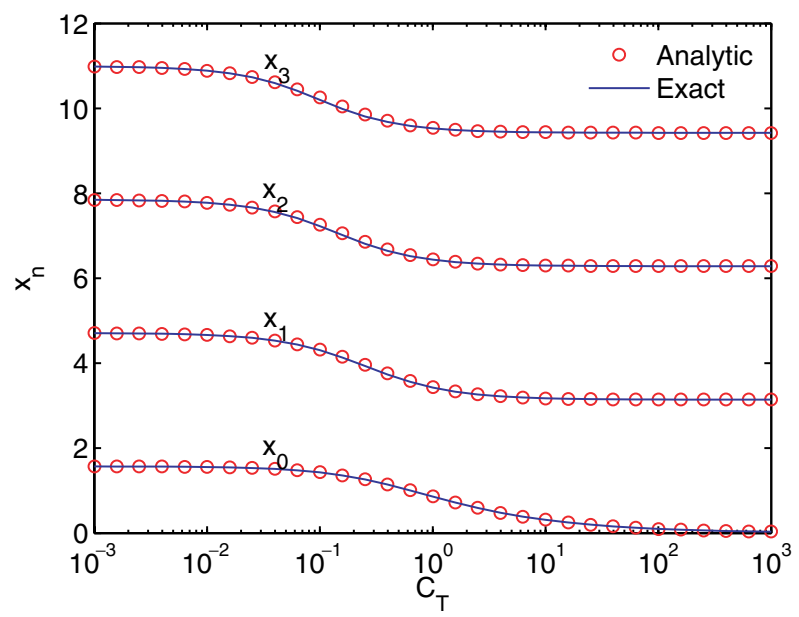

Fig. 4. Analytic solution of Eq. (13) as compared with the exact solution for different values of $C_{T}$.

The residue of the corresponding poles $k_{n, \pm}$ can be solved as

$$
\begin{aligned}
k_{n, \pm} & =\lim _{s \rightarrow p_{n, \pm}} \frac{s-p_{n, \pm}}{F(s)}=\frac{1}{F^{\prime}\left(p_{n, \pm}\right)} \\
& =\frac{ \pm 2 x_{n}}{\sqrt{R^{2} C^{2}-4 L C x_{n}^{2}}\left[\left(1+C_{T}\right) \sin x_{n}+C_{T} x_{n} \cos x_{n}\right]}
\end{aligned}
$$

\subsection{Step and ramp response}

From the poles and corresponding residues, the transfer function can be represented as

$$
H(s)=\sum_{i} \frac{k_{i}}{s-p_{i}},
$$

where $i$ is the index covering all of the poles. Consider a wire structure example as shown in Fig. 5. The interconnect parameters per unit length are $R_{\text {int }}=$ $12.24 \mathrm{~m} \Omega / \mu \mathrm{m}, L_{\text {int }}=0.74 \mathrm{pH} / \mu \mathrm{m}$, and $C_{\text {int }}=0.266 \mathrm{fF} / \mu \mathrm{m}$, which are extracted from FastHenry ${ }^{18}$ and FastCap ${ }^{19}$ with a signal frequency of $2 \mathrm{GHz}$. The amplitude of the transfer function obtained from Eq. (20) is compared with the exact transfer

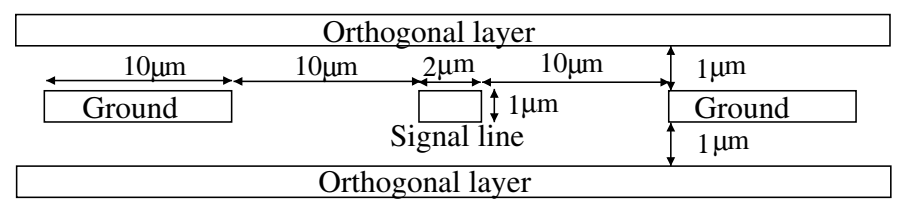

Fig. 5. Wire geometry of an example circuit, where the signal wire is shielded by two ground lines. 


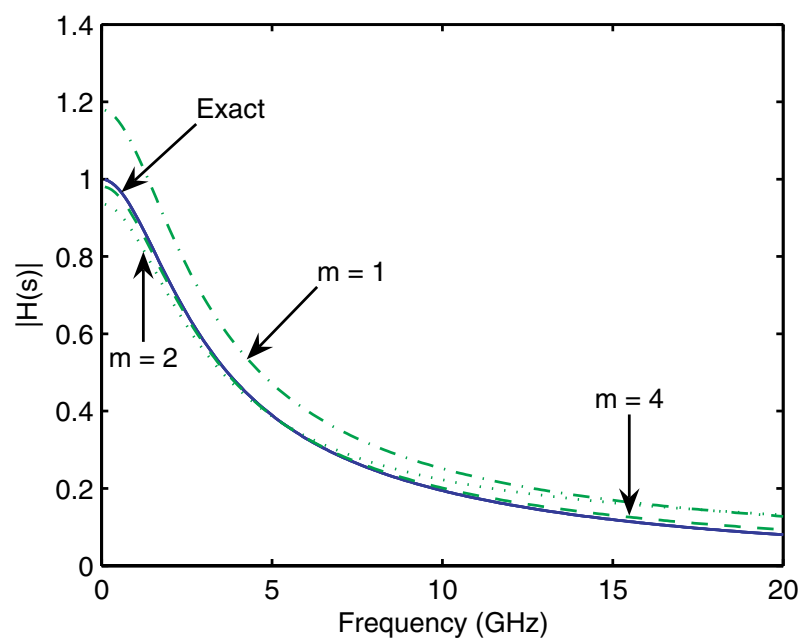

(a)

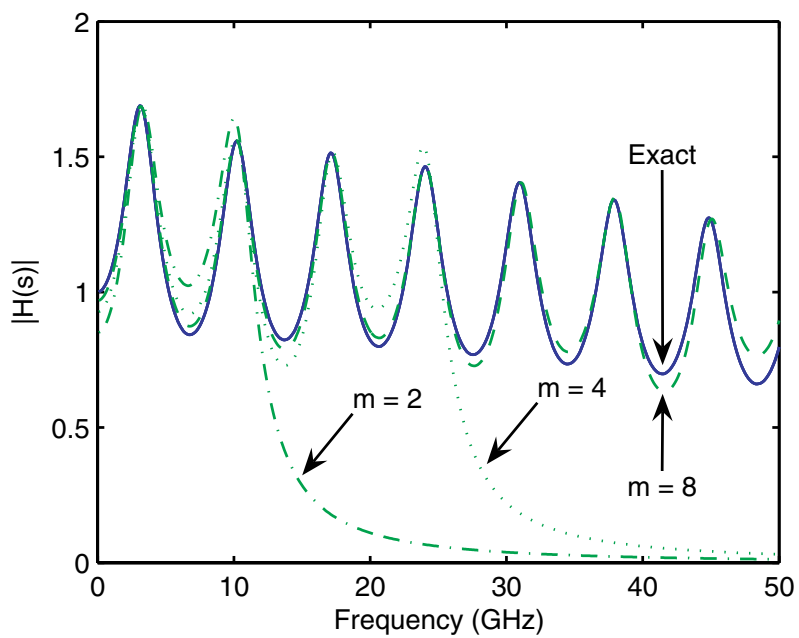

(b)

Fig. 6. Comparison between the analytic expression (20) and the exact transfer function. The wire length is $5 \mathrm{~mm}$ and the load capacitance is $C_{L}=50 \mathrm{fF}$; (a) $R C$ interconnect case, $R_{d}=30 \Omega$. (b) $R L C$ interconnect with a zero $R_{d}$.

function for the $R C$ case in Fig. 6(a) and $R L C$ case with a zero $R_{d}$ in Fig. 6(b), respectively. In Fig. 6(a), $m$ is the number of poles considered in the model. In Fig. 6(b), $m$ is the number of pole pairs, since the poles in this case are in pairs. As shown in the figure, the analytic transfer function converges to the exact transfer function with increasing $m$. As compared with the $R C$ case, more poles are required for the $R L C$ case to obtain an accurate result. 
From Eq. (20), the normalized step response $V_{s}(t) / V_{d d}$ and ramp response $V_{r}(t) / V_{d d}$ are, respectively,

$$
\begin{aligned}
& \frac{V_{s}(t)}{V_{d d}}=u(t)\left[1+\sum_{i}\left(\frac{k_{i}}{p_{i}} e^{p_{i} t}\right)\right], \\
& \frac{V_{r}(t)}{V_{d d}}=V_{1}(t)-V_{1}\left(t-t_{r}\right), V_{1}(t)=\frac{u(t)}{t_{r}}\left[t+m_{1}+\sum_{i}\left(\frac{k_{i}}{p_{i}^{2}} e^{p_{i} t}\right)\right],
\end{aligned}
$$

where $u(t)$ is the step function. The following moment information is used,

$$
m_{0}=-\sum_{i} \frac{k_{i}}{p_{i}}=1, \quad m_{1}=-\sum_{i} \frac{k_{i}}{p_{i}^{2}} .
$$

For an $R C$ interconnect,

$$
m_{1}=-R_{d}\left(C+C_{L}\right)-R\left(0.5 C+C_{L}\right),
$$

and for an $R L C$ interconnect with a zero driver resistance,

$$
m_{1}=-R\left(0.5 C+C_{L}\right) .
$$

The step and ramp responses obtained from Eqs. (21) and (22) are compared with Spectre simulations in Fig. 7. In the Spectre simulation, the transmission line is modeled as a series of $\pi$-shaped $R C$ or $R L C$ segments. Each segment is $10 \mu \mathrm{m}$ long. Good agreement between the analytic solution and Spectre simulations is observed. The accuracy of the ramp response is much higher than that of the step response since a ramp signal consists of fewer high frequency components.

\section{Distributed $R L C$ Interconnect with Driver Resistance}

For an interconnect driven by a gate, there are primarily two kinds of approaches for timing analysis. In the first approach, the driver and the interconnect are separated. The voltage waveform at the gate output is obtained through pre-characterized delay and transition time information characterizing the gate. ${ }^{20}$ This waveform is applied at the input of the interconnect to obtain the far end response. With increasing inductive effects, more complicated driver output models are required to characterize the reflection behavior of the propagating signals, such as the tworamp model described in Ref. 21 and the three-piece model in Ref. 22. Recently, several current source models (CSM) have been developed, ${ }^{23-25}$ where the nonlinear behavior of the gate is characterized, making the driver output response more accurate. In the second approach, the driver and interconnect are analyzed as a single system, where the Thevenin model is generally used, 5, 7,10,26,27 as shown in Fig. 1. In this approach, the interaction between the driver and interconnect is modeled as a single system.

For the first approach, once the driver output voltage is obtained, this voltage waveform can be treated as a voltage source with zero resistance, and applied to 


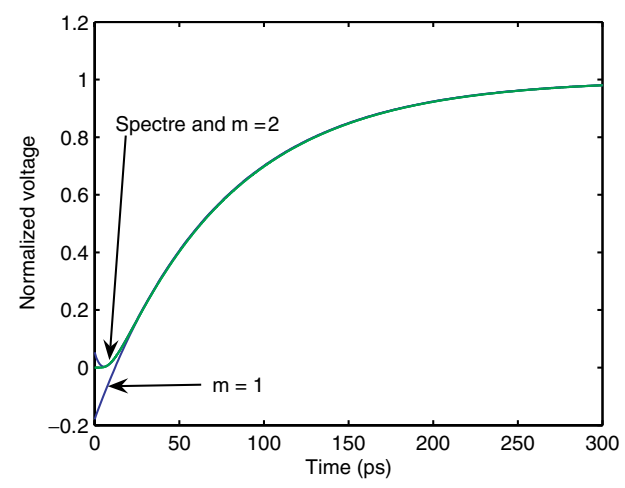

(a) Step response, $R C$

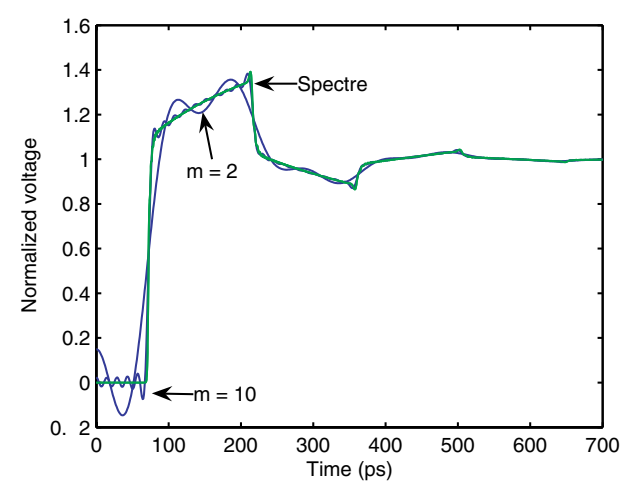

(c) Step response, $R L C$

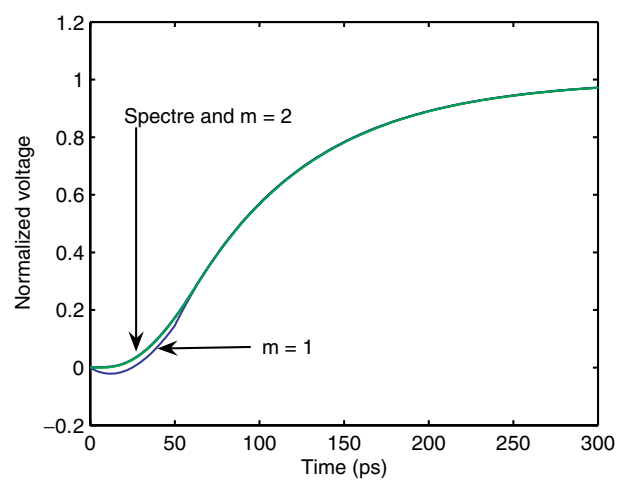

(b) Ramp response, $R C, t_{r}=50 \mathrm{ps}$

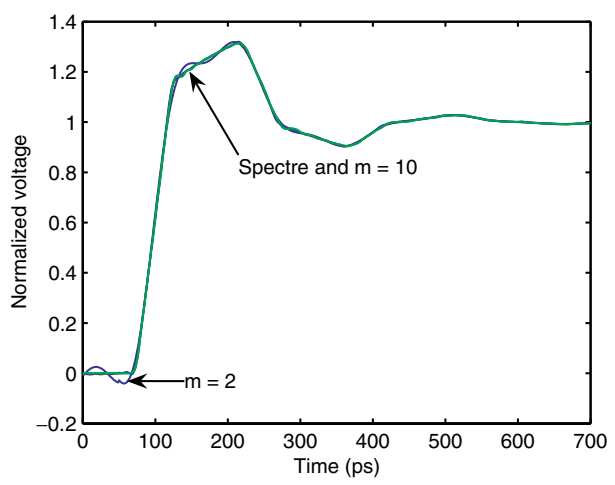

(d) Ramp response, $R L C, t_{r}=50 \mathrm{ps}$

Fig. 7. Step and ramp response obtained analytically as compared with Spectre simulations. (a) Step response, $R C$, (b) Ramp response, $R C$, (c) Step response, $R L C$, and (d) Ramp response, $R L C$.

the input of an interconnect. By representing this voltage source as a piecewise linear waveform, the far end response is a combination of a number of ramp and/or step responses, which are solved in Sec. 2.2. For the second voltage approach, the method proposed in Sec. 2.2 needs to be improved to include the effect of the driver resistance. With a system transform, the poles of a general $R L C$ interconnect system are solved in Sec. 3.1. The accuracy of the poles are further improved with the Newton-Raphson method as described in Sec. 3.2. The accuracy and efficiency of the proposed model are discussed in Sec. 3.3.

\subsection{System transform}

In Ref. 9, the circuit model as shown in Fig. 1 is mapped into an open-ended interconnect system by matching the moments. Similarly, the interconnect system with a driver resistance can also be mapped into a system without a driver resistance. 
Consider a step signal at the input of the circuit shown in Fig. 1. The height of the initial step at the driver output is $V_{d d} Z_{0} /\left(R_{d}+Z_{0}\right)$, where $Z_{0}=\sqrt{L / C}$ is the characteristic impedance of a lossless line. As described in Ref. 28, the attenuation coefficient of a transmission line saturates with increasing frequency to the asymptotic value $R / 2 Z_{0}$. Assume the total interconnect resistance of the new system (without a driver resistance) is $R^{\prime}$ and the load capacitance is $C_{L}^{\prime}$. By matching the amplitude of the initial propagating wave,

$$
V_{d d} \frac{Z_{0}}{R_{d}+Z_{0}} e^{-\frac{R}{2 Z_{0}}}=V_{d d} e^{-\frac{R^{\prime}}{2 Z_{0}}},
$$

$R^{\prime}$ can be obtained as

$$
R^{\prime}=R+2 Z_{0} \log \left(1+\frac{R_{d}}{Z_{0}}\right) .
$$

By matching the first moment of the two systems,

$$
-m_{1}=R_{d}\left(C_{L}+C\right)+R\left(0.5 C+C_{L}\right)=R^{\prime}\left(0.5 C+C_{L}^{\prime}\right),
$$

$C_{L}^{\prime}$ can be obtained as

$$
C_{L}^{\prime}=\frac{-m_{1}}{R^{\prime}}-0.5 C
$$

After this conversion, the method proposed in Sec. 2.2 can be applied. In Fig. 8, the waveform obtained from the proposed model is compared with Spectre simulations and another four-pole model described in Ref. 6. This four-pole model is obtained by truncating the denominator of the transfer function to the fourth-order; however, no closed-form solution is available for solving the four poles. Note that although both the proposed model and the four-pole model are based on an approximation of the four poles of the system, the proposed model is much more accurate than the four-pole model when inductive effects are important (a system with a small driver resistance), as shown in Fig. 8(a). When the system is dominated by the driver resistance, the proposed model is less accurate, particularly at the beginning period of the waveform, as shown in Fig. 8(b).

\subsection{Improve the accuracy of the poles}

The location of the low order poles obtained analytically is compared with the location of the exact poles in Fig. 9. From the figure, note that there is a one-toone mapping between the approximated poles and the exact poles. The real pole without an arrow in Fig. 9 is mapped to a real pole which is out of the range of the figure. From these approximated poles, the exact poles are obtained through the Newton-Raphson method, permitting the accuracy of the model to be significantly improved. In general, the number of iterations required for convergence is less than five.

Special attention needs to be paid to those real poles when applying the NewtonRaphson method. For example, the Newton-Raphson process starting from the 


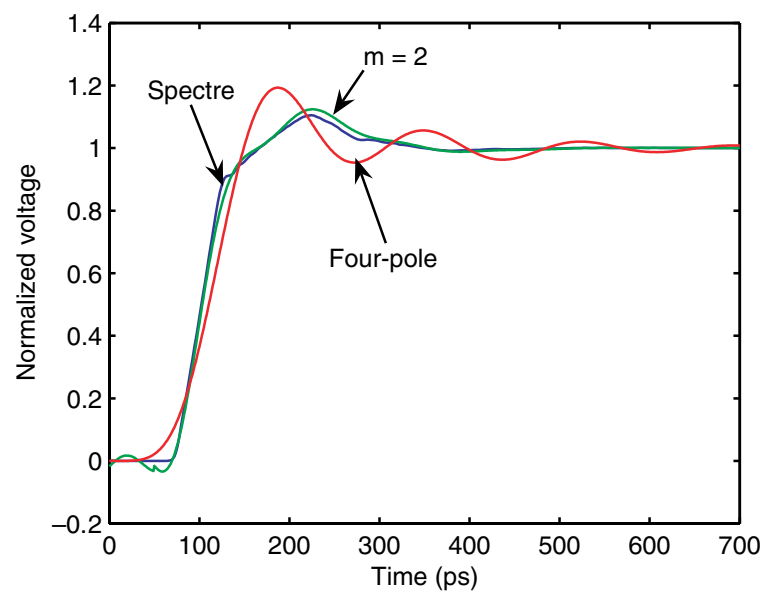

(a)

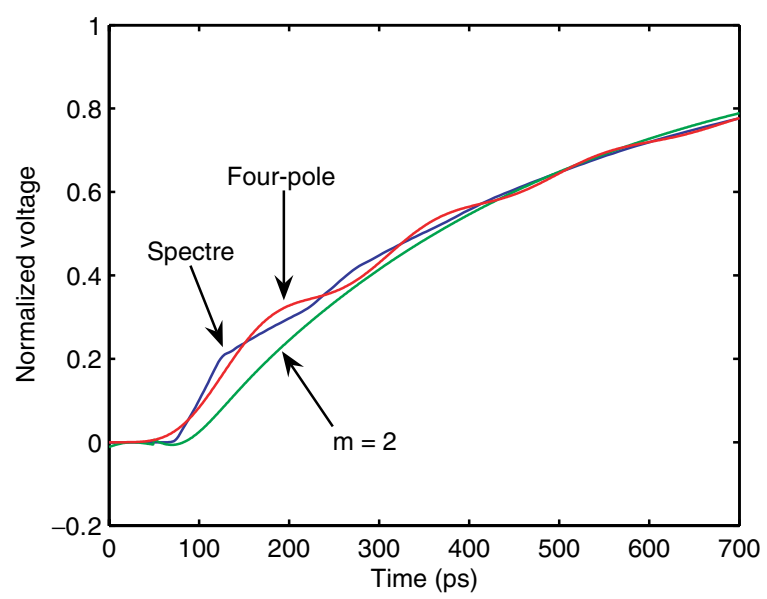

(b)

Fig. 8. Transient response of a transmission line obtained with the proposed model, four-pole model, and Spectre simulations. $t_{r}=50 \mathrm{ps}$ and $C_{L}=50 \mathrm{fF}$. (a) $R_{d}=20 \Omega$, (b) $R_{d}=300 \Omega$.

approximated pole $-3.892 \times 10^{10}$ (the left real pole as shown in Fig. 9) incorrectly converges to the exact pole $-6.396 \times 10^{9}$ rather than converges to the exact pole outside the range of the figure. In order to distinguish this case from the double real pole case, the following condition needs to be evaluated. If $p$ is a double real pole of the system, $p$ satisfies the following expression,

$$
\lim _{s \rightarrow p} \frac{F(s)}{s-p}=F^{\prime}(p)=0 .
$$

For systems with multiple real poles, the system is dominated by the real pole with the smallest magnitude and the effect of the other real poles can be ignored, 


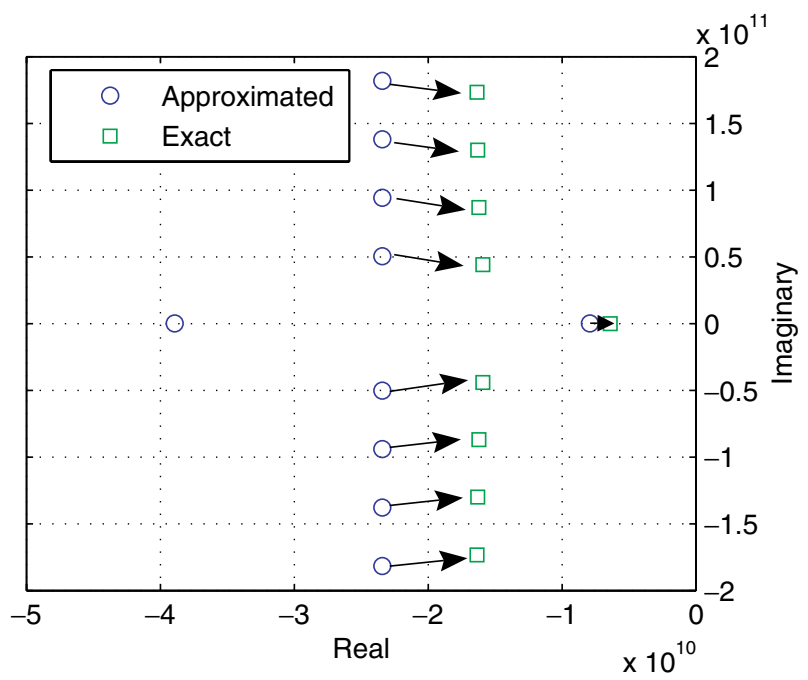

Fig. 9. Mapping between the approximated poles and the exact poles, $R_{d}=100 \Omega$.

unless these poles are close to the dominant pole. The distance between the other real poles and the dominant real pole is related to the value of $F^{\prime}\left(p_{d}\right)$, where $p_{d}$ is the dominant pole. If there is another pole $p_{x}$ which is close to $p_{d}, F^{\prime}\left(p_{d}\right)$ should be small. When $p_{x}$ approaches $p_{d}$, the value of $F^{\prime}\left(p_{d}\right)$ approaches zero. In the limit, $p_{x}=p_{d}, p_{d}$ is a double pole, and $F^{\prime}\left(p_{d}\right)=0$, as expressed in Eq. (30).

Pseudo-code for generating the exact poles of a single interconnect system is shown in Fig. 10. In Fig. 10, the variable over_damped is used to indicate whether the system is overdamped or not. For overdamped systems, the higher order real poles (with $n>0$ ) are ignored. A threshold value $F_{t h}$ is set for $F^{\prime}(p)$, which is used to indicate the distance between other high order real poles and the dominant real pole. After the dominant real pole (if the system has real poles, the dominant real pole is always $\left.p_{0,+}\right)$ is determined, $F^{\prime}\left(p_{0,+}\right)$ is evaluated. $F(s)$ can be represented by the poles as

$$
F(s)=\prod_{n=0}^{\infty}\left(1-\frac{s}{p_{n,+}}\right)\left(1-\frac{s}{p_{n,-}}\right) .
$$

From Eq. (31),

$$
\begin{aligned}
F^{\prime}\left(p_{0,+}\right) & =\frac{-1}{p_{0,+}}\left(1-\frac{p_{0,+}}{p_{0,-}}\right) \prod_{n=1}^{\infty}\left(1-\frac{p_{0,+}}{p_{n,+}}\right)\left(1-\frac{p_{0,+}}{p_{n,-}}\right) \\
& <\frac{-1}{p_{0,+}}\left(1-\frac{p_{0,+}}{p_{0,-}}\right) .
\end{aligned}
$$

If $\left|p_{0,-}\right|>2\left|p_{0,+}\right|, F^{\prime}\left(p_{0,+}\right)<-0.5 / p_{0,+}$. With some guardband, $F_{t h}$ is determined as $-0.3 / p_{0,+}$. If $F^{\prime}\left(p_{0,+}\right)<F_{t h}$, which means pole $p_{0,-}$ is close to $p_{0,+}$, a 
Input: $R, L, C, R_{d}, C_{L}$

Output: $m$ pairs of low-order poles

Find_poles $\left(R, L, C, R_{d}, C_{L}\right)$

\{

calculate $R^{\prime}$ and $C_{L}^{\prime}$

calculate $m$ pairs of approximated poles $p_{n, \pm}$

over_damped $=0$

for $n=0: m-1$

\{

$p_{n, \pm}=$ Newton_Raphson $\left(p_{n, \pm}\right)$

if $p_{n,+}$ is real

if over_damped $==1$

else

discard poles $p_{n, \pm}$

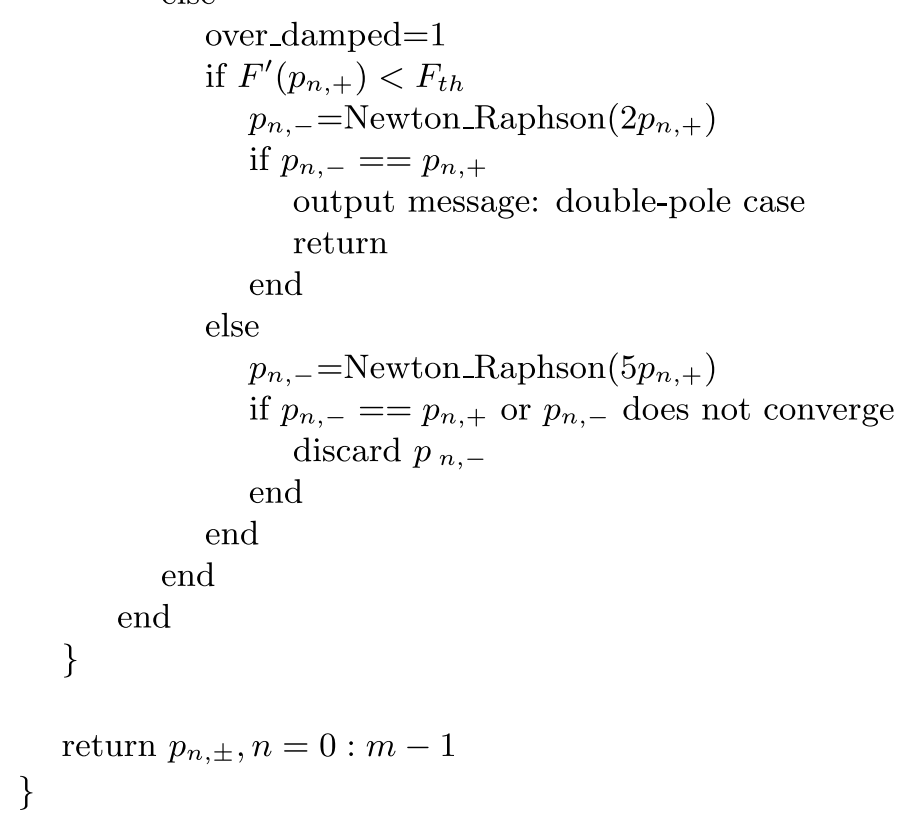

Fig. 10. Pseudo-code for computing the exact poles. The function Newton_Raphson( ) is the Newton-Raphson converging process starting with the input argument.

Newton_Raphson process is launched from point $2 p_{0,+}$ to determine $p_{0,-}$. Otherwise, the Newton_Raphson process is launched from point $5 p_{0,+}$ to determine $p_{0,-}$. If the process does not converge or incorrectly converges to $p_{0,+}$, which means the true value of $\left|p_{0,-}\right|$ is greater than $5\left|p_{0,+}\right|$, the effect of $p_{0,-}$ can be ignored. For the double pole case, the process of solving the residue requires the complicated process of solving the second order derivative of $F(s)$. The code produces an output message if a double pole occurs. In this case, a small change in the circuit parameters 
can avoid a double pole, while the effect on the output signal waveform caused by this parameter change cannot be distinguished. After the exact poles are extracted, a step or ramp response is constructed from Eqs. (21) or (22). In order to eliminate the artificial discontinuity of the waveform at the end of the input rising edge, the first moment $m_{1}$ in Eq. (22) is calculated from the truncated summation, as shown in the left side of Eq. (23), rather than the exact value of $0.5 R^{\prime} C+R^{\prime} C_{L}^{\prime}$.

For the same circuit examples used in Fig. 8, the waveform obtained from the improved method is re-plotted in Fig. 11. From Fig. 11, the difference between the analytic waveforms and Spectre simulations is difficult to distinguish except for the period of the initial time-of-flight.

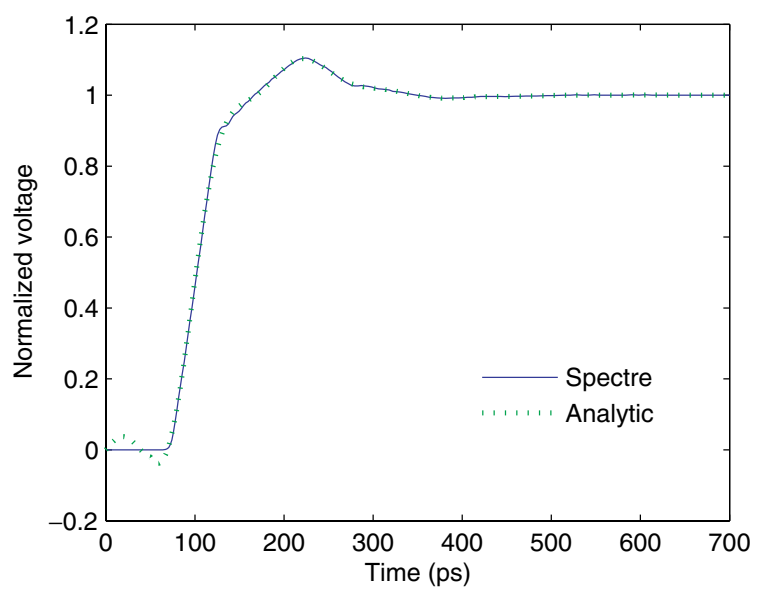

(a)

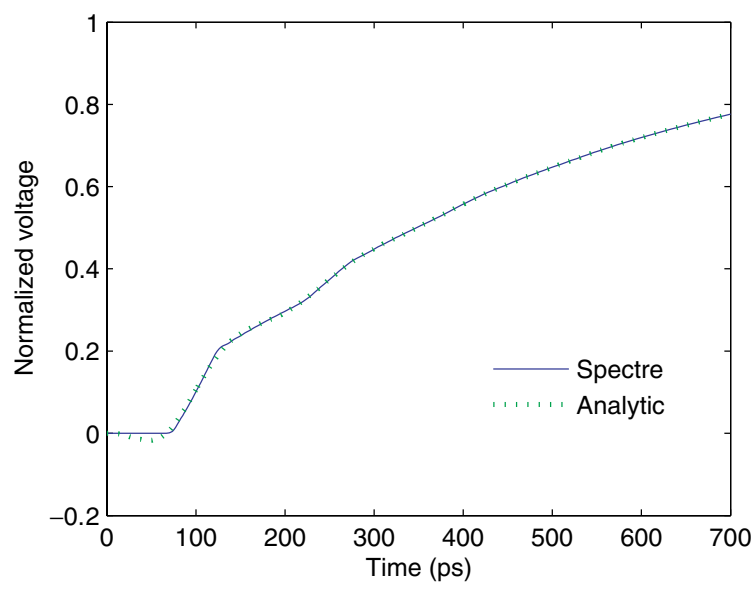

(b)

Fig. 11. Transient response of transmission line obtained with the improved analytic method as compared with Spectre simulations, $m=2$, (a) $R_{d}=20 \Omega$, (b) $R_{d}=300 \Omega$. 


\subsection{Model accuracy and efficiency}

The $50 \%$ delay, $10 \%$-to-90\% rise time, and the normalized overshoot obtained from the proposed model are compared in Fig. 12 with Spectre simulations for different input rise times (the input rise time is determined from 0 to $V_{d d}$ ). Since the signal delay is generally determined by the low frequency components, two pairs of poles provide a sufficiently accurate delay estimation. The average error is $1 \%$ for different input rise times. For the output rise time and overshoot, the error is larger for smaller input rise times. The error decreases with increasing input rise time,

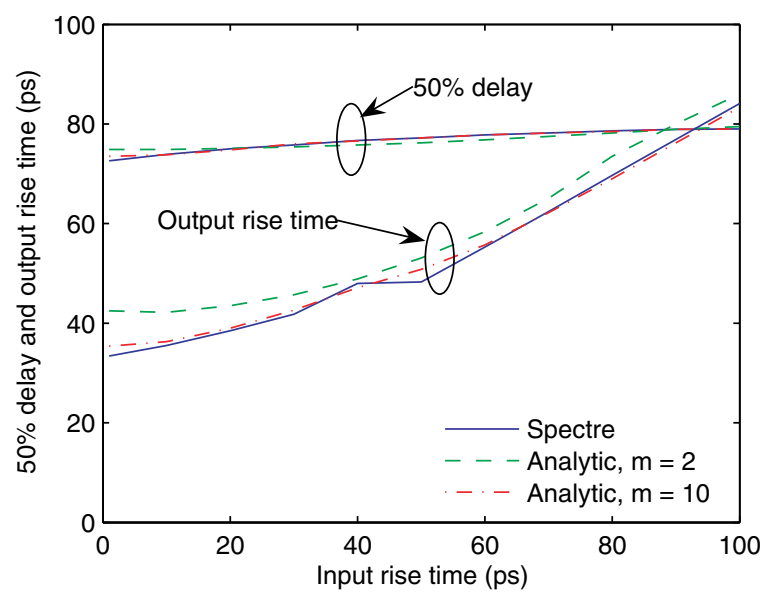

(a)

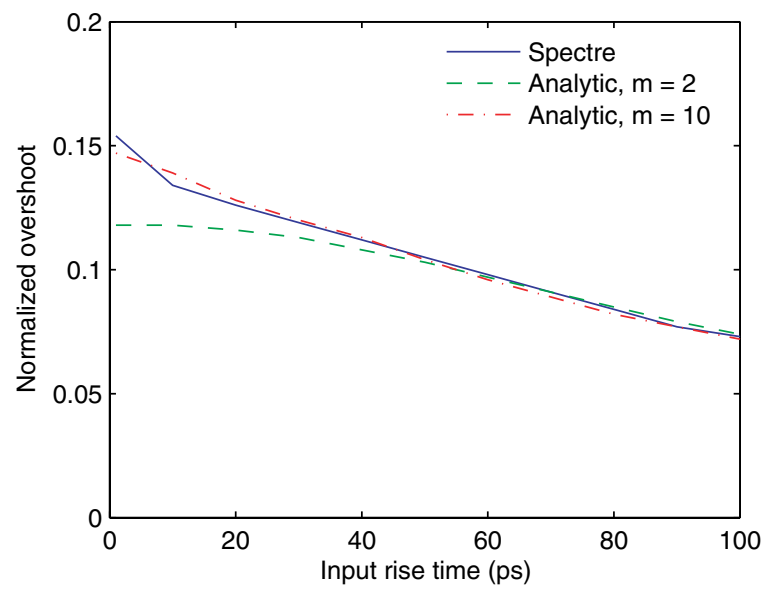

(b)

Fig. 12. Comparison of the $50 \%$ delay, $10 \%$-to- $90 \%$ output rise time, and the normalized overshoot obtained from the proposed model and Spectre simulations, $R_{d}=20 \Omega, C_{L}=50 \mathrm{fF}$, and $l=5 \mathrm{~mm}$; (a) Delay and output rise time, (b) Overshoot. 
since the output rise time and overshoot are closely related to the high frequency components. The average error with two pairs of poles is $9.5 \%$ for the output rise time and $5.5 \%$ for the overshoot. When the number of pole pairs increases to ten, these two average errors decrease to $2.0 \%$ and $1.9 \%$, respectively.

The computational complexity of the proposed method is approximately proportional to the number of pole pairs. These experiments have been performed on a SunBlade1500 workstation. The time required for Spectre to perform a $700 \mathrm{ps}$ transient simulation (250 time steps) is $1.8 \mathrm{~s}$. The proposed model is implemented with Matlab. The run time is $3.1 \mathrm{~ms}$ for $m=2$ and $10.9 \mathrm{~ms}$ for $m=10$. To achieve an accuracy similar to the proposed model $(m=2)$, more than 12 poles are required in the traditional moment matching method. Since there are no closed-form solutions for solving the poles from the moments, the computational complexity of the moment matching method is higher as compared with the proposed method. Specifically, the run time for the moment matching method with 12 poles is $13.5 \mathrm{~ms}$ as compared to $3.1 \mathrm{~ms}$ for the proposed method with $m=2$. Furthermore, the moment matching method suffers numerical stability problems with high order approximations. The accuracy of the proposed model is also verified for different interconnect lengths and is illustrated in Fig. 13.

\section{Frequency Dependent Effects}

Both interconnect inductance and resistance are a function of frequency. This frequency dependent interconnect impedance affects the signal waveform, particularly for those signals containing a greater number of high frequency components.

From Eq. (27), the contribution of the driver resistance to the effective interconnect resistance is $R_{d_{-} e f f}=2 Z_{0} \log \left(1+R_{d} / Z_{0}\right)$, which is frequency independent (the frequency dependence due to $Z_{0}$ is ignored, and the $Z_{0}$ used here is determined at DC). The effective load capacitance is also determined at DC, as shown in Eq. (29). Considering the effect of the driver resistance and the frequency dependence of $R$ and $L$ of the interconnect, the effective propagation coefficient $\theta$ becomes

$$
\theta=\sqrt{\left[R_{d_{-} e f f}+R(s)+L(s) s\right] C s} .
$$

For different functional forms of $R(s)$ and $L(s)$, the poles of the transfer function of an interconnect can be obtained by solving Eq. (33). Closed-form solutions may also be available depending upon the expressions of $R(s)$ and $L(s)$.

The frequency dependent impedance can be modeled by ladder structures of frequency-independent elements. ${ }^{29,30}$ These ladder structures are particularly suitable to capture skin effects. A two stage ladder structure ${ }^{29}$ is adopted in this paper for simplicity, as shown in Fig 14. Since the frequency dependent effect is naturally more significant at high frequencies, a wider interconnect is adopted so that additional high frequency components can propagate across the interconnect, distinguishing the frequency dependent effects. The signal wire width is $10 \mu \mathrm{m}$, the space 


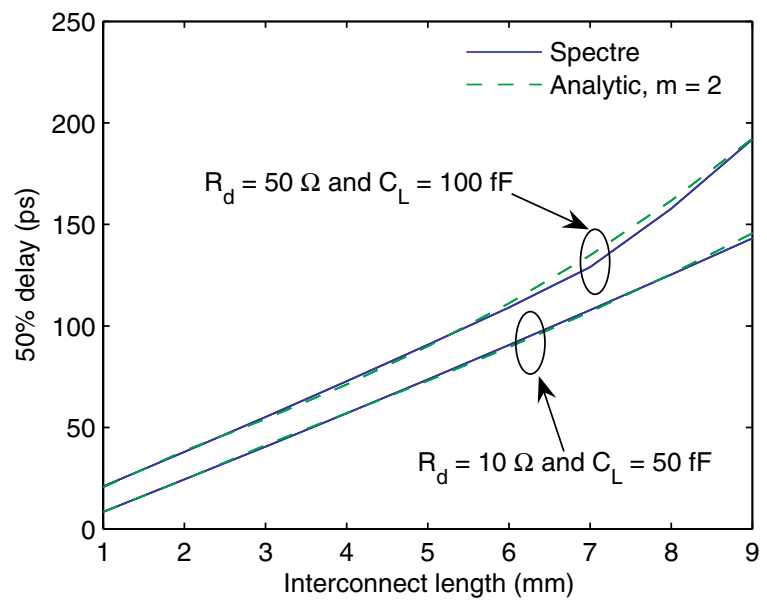

(a)

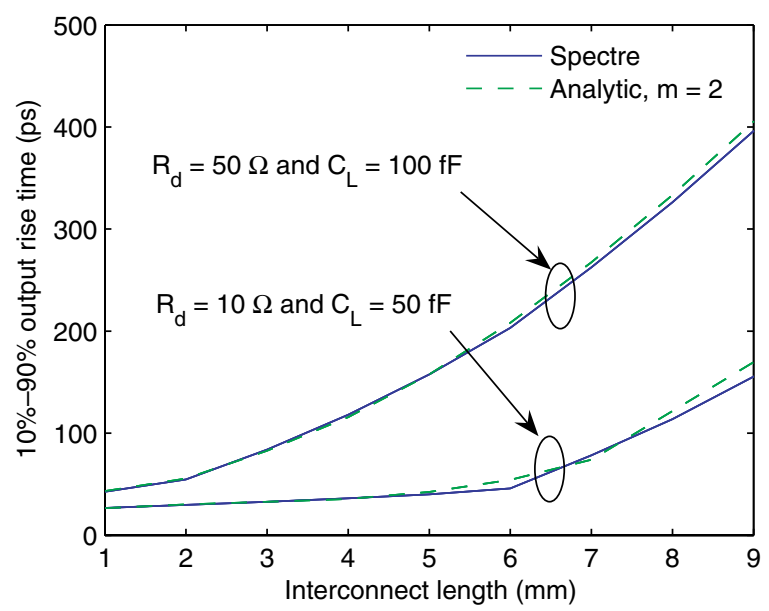

(b)

Fig. 13. Comparison of the $50 \%$ delay and $10 \%$-to- $90 \%$ output rise time obtained from the proposed model and Spectre simulations; $t_{r}=50$ ps, (a) $50 \%$ delay, (b) $10 \%$-to- $90 \%$ output rise time.
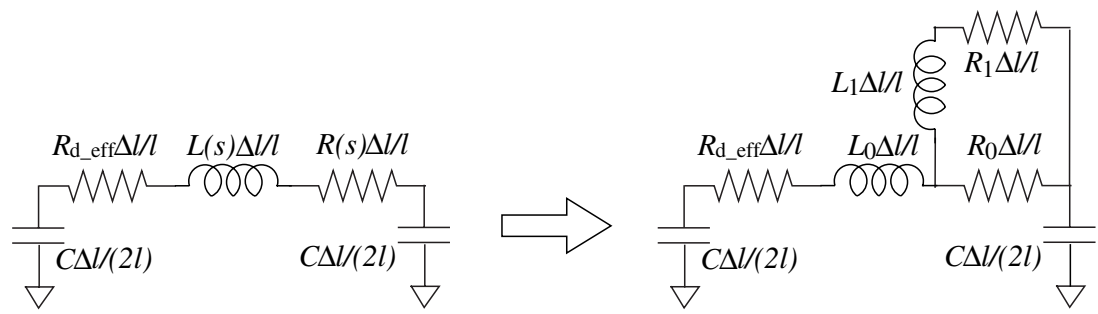

Fig. 14. A segment of interconnect with length $\Delta l$. 
between the signal line and ground is $5 \mu \mathrm{m}$, and the remaining geometric parameters are the same as depicted in Fig. 5. The parameters in the ladder structure are calculated by matching the DC and high frequency resistance and inductance of the ladder structure with the extracted values. Since the resistance of the interconnect does not saturate at high frequencies, a value of $40 \Omega$ is assumed as the high frequency resistance in this example, resulting in the following parameters: $R_{0}=40 \Omega, R_{1}=28.1 \Omega, L_{0}=1.9 \mathrm{nH}$, and $L_{1}=1.12 \mathrm{nH}$. The DC impedance is $R_{d c}=16.5 \Omega, L_{d c}=2.287 \mathrm{nH}$, and $C=4.18 \mathrm{pF}$. The resistance and inductance of the ladder approximation are compared with the extracted values in Fig. 15.

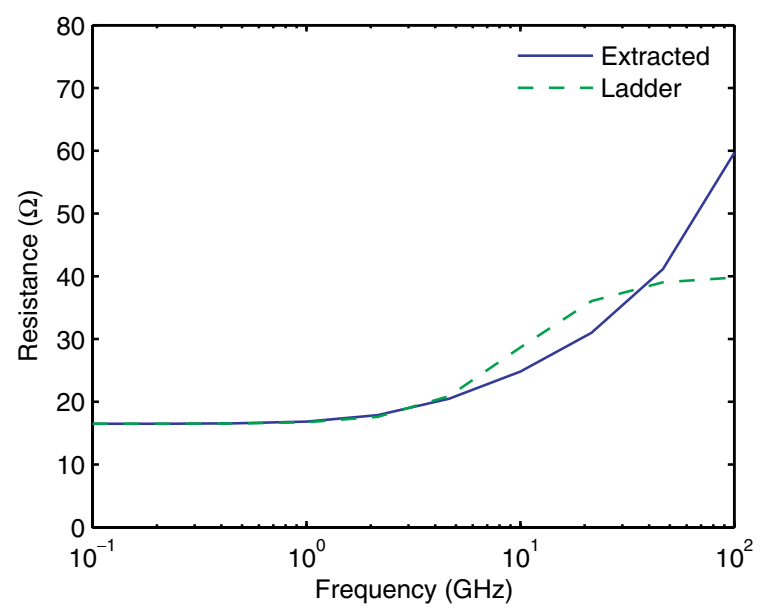

(a)

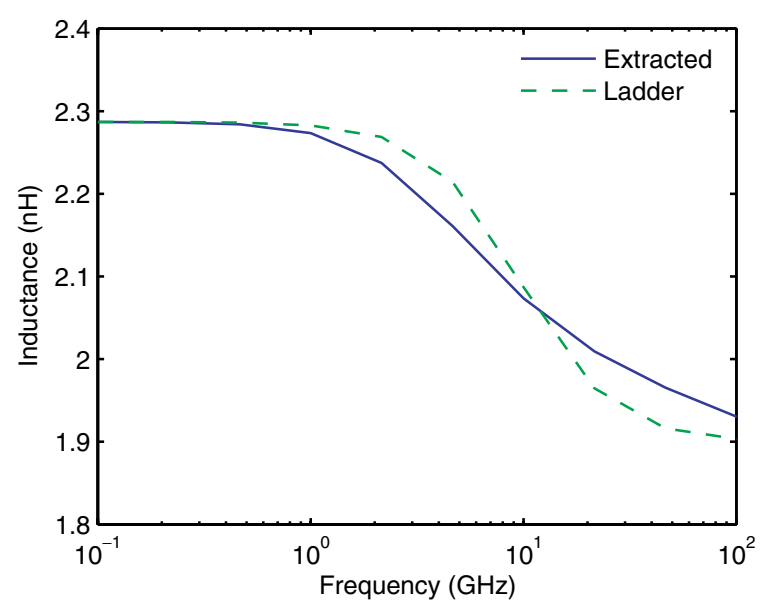

(b)

Fig. 15. Frequency dependent impedance of an interconnect with a length of $5 \mathrm{~mm}$; (a) Resistance, (b) Inductance. 
With this ladder approximation, the expression used to solve the poles of the system becomes

$$
\left[R_{d \_e f f}+L_{0} s+\frac{R_{0}\left(R_{1}+L_{1} s\right)}{R_{0}+R_{1}+L_{1} s}\right] C s=\theta^{2}=-x_{n}^{2} .
$$

The poles can be analytically solved as

$$
p_{n, \pm}=-\frac{a_{2}}{3}-\frac{X}{2} \pm \frac{\sqrt{3}}{2} i \sqrt{X^{2}+4 Q}
$$

where

$$
\begin{aligned}
& Q=\frac{3 a_{1}-a_{2}^{2}}{9}, \\
& P=\frac{9 a_{1} a_{2}-27 a_{0}-2 a_{2}^{3}}{54}, \\
& X=\sqrt[3]{P+\sqrt{Q^{3}+P^{2}}}+\sqrt[3]{P-\sqrt{Q^{3}+P^{2}}}
\end{aligned}
$$

and

$$
\begin{aligned}
a_{2} & =\frac{L_{0} R_{0}+L_{0} R_{1}+R_{0} L_{1}+L_{1} R_{d_{-} e f f}}{L_{0} L_{1}}, \\
a_{1} & =\frac{R_{0} R_{1} C+\left(R_{0}+R_{1}\right) R_{d_{-} e f f} C+x_{n}^{2} L_{1}}{L_{0} L_{1} C}, \\
a_{0} & =\frac{\left(R_{0}+R_{1}\right) x_{n}^{2}}{L_{0} L_{1} C} .
\end{aligned}
$$

From Eq. (35), the Newton-Raphson method can be applied to solve the exact poles as illustrated in Sec. 3.2. In Fig. 16, the output signal waveforms are compared for the DC impedance case and the frequency dependent (FD) impedance case.

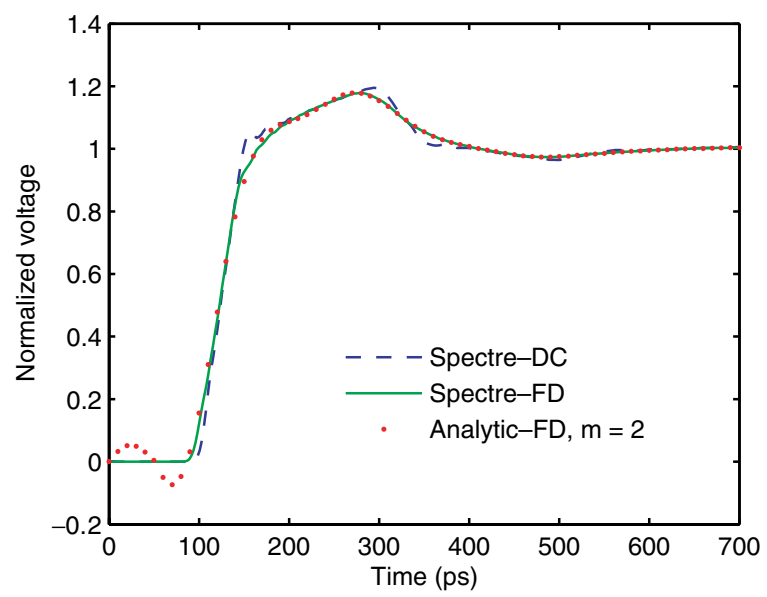

Fig. 16. Comparison of the output signal waveforms with and without the frequency dependent effect, $R_{d}=10 \Omega, C_{L}=50 \mathrm{pF}$, and $t_{r}=50 \mathrm{ps}$. 


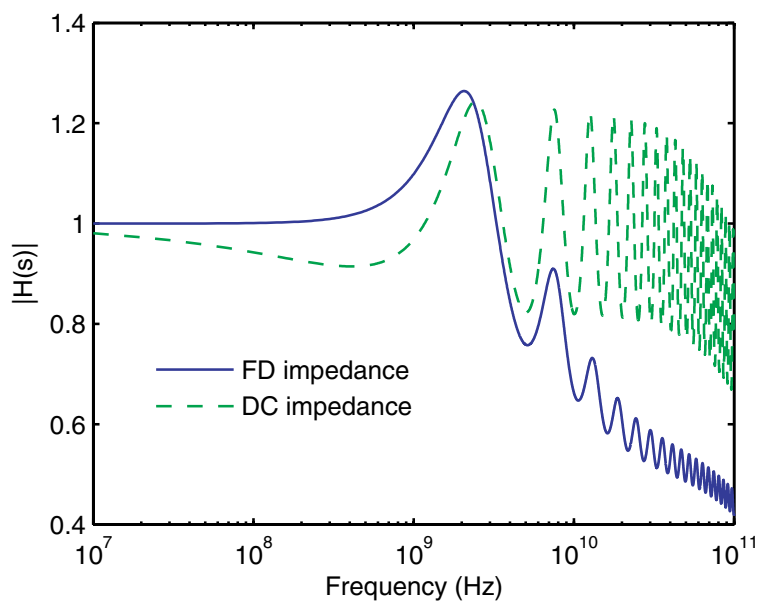

Fig. 17. Comparison of transfer functions with and without the frequency dependent effect, $R_{d}=10 \Omega$ and $C_{L}=50 \mathrm{pF}$.

As shown in Fig. 16, by considering the FD effect, additional high frequency components are suppressed, making the waveform smoother since the high frequency components experience much greater attenuation due to the increasing interconnect resistance, as shown in Fig. 17. For the high frequency related waveform properties, such as the rise time and overshoot, the FD effect should be considered. For low frequency related waveform properties, such as delay, the FD effect can be neglected. Similar results are also described in Ref. 31. The run time of the Spectre simulation ( $700 \mathrm{ps}, 225$ time steps) is $2.45 \mathrm{~s}$ and the run time for the proposed analytic method $(m=2)$ is $3.8 \mathrm{~ms}$ (three orders of magnitude improvement in computational time).

\section{Conclusions}

By extracting the exact poles, an efficient method has been proposed in this paper for determining the transient output response of a distributed $R L C$ interconnect. As demonstrated in the paper, two pairs of poles can provide an accurate delay estimate exhibiting an average error of $1 \%$ as compared with Spectre simulations. For high frequency related waveform properties, such as the rise time and overshoot, an average error of less than $2 \%$ can be obtained with ten pairs of poles. The computational complexity of the proposed method is proportional to the number of pole pairs. By using a ladder structure, frequency dependent effects can also be included in the method. Excellent agreement is observed between the proposed model and Spectre simulations.

\section{References}

1. V. V. Deodhar and J. A. Davis, Optimal voltage scaling, repeater insertion, and wire sizing for wave-pipelined global interconnects, IEEE Trans. Circuits Syst. I $5 \mathbf{5}$ (2008) $1023-1030$. 
2. S. Y. Kim and S. S. Wong, Closed-form RC and RLC delay models considering input rise time, IEEE Trans. Circuits Syst. I 54 (2007) 2001-2010.

3. T. Sakurai, Approximation of wiring delay in MOSFET LSI, IEEE J. Solid-State Circuits 18 (1983) 418-426.

4. T. Sakurai, Closed-form expressions for interconnection delay, coupling, and crosstalk in VLSI's, IEEE Trans. Electron. Dev. 40 (1993) 118-124.

5. A. B. Kahng and S. Muddu, An analytical delay model for $R L C$ interconnects, IEEE Trans. Comput.-Aided Des. 16 (1997) 1507-1514.

6. K. Banerjee and A. Mehrotra, Accurate analysis of on-chip inductance effects and implications for optimal repeater insertion and technology scaling, Proc. IEEE Symp. VLSI Circuits, June 2001, pp. 195-198.

7. J. A. Davis and J. D. Meindl, Compact distributed $R L C$ interconnect models Part I: Single line transient, time delay, and overshoot expressions, IEEE Trans. Electron Dev. 47 (2000) 2068-2077.

8. Y. Eo, J. Shim and W. R. Eisenstadt, A traveling-wave-based waveform approximation technique for the timing verification of single transmission lines, IEEE Trans. Comput.-Aided Des. 21 (2002) 723-730.

9. J. Chen and L. He, Piecewise linear model for transmission line with capacitive loading and ramp input, IEEE Trans. Comput.-Aided Des. 24 (2005) 928-937.

10. G. Chen and E. G. Friedman, An $R L C$ interconnect model based on Fourier analysis, IEEE Trans. Comput.-Aided Des. 24 (2005) 170-183.

11. W. J. Dally and B. Towles, Route packets, not wires: On-chip interconnection networks, Proc. IEEE/ACM Design Automation Conf., June 2001, pp. 684-689.

12. Y. Cao et al., Effective on-chip inductance modeling for multiple signal lines and application to repeater insertion, IEEE Trans. VLSI Syst. 10 (2002) 799-805.

13. L. T. Pillage and R. A. Rohrer, Asymptotic waveform evaluation for timing analysis, IEEE Trans. Comput.-Aided Des. 9 (1990) 352-366.

14. P. Feldmann and R. W. Freund, Efficient linear circuit analysis by Pade approximation via the Lanczos process, IEEE Trans. Comput.-Aided Des. 14 (1995) 639-649.

15. M. Silveria, M. Kamon and J. White, Efficient reduced-order modeling of frequencydependent coupling inductances associated with 3-D interconnect structures, IEEE Trans. Comp., Packag. Manuf. Technol. B 19 (1996) 283-288.

16. A. Odabasioglu, M. Celik and L. T. Pillage, PRIMA: Passive reduced-order interconnect macromodeling algorithm, IEEE Trans. Comput.-Aided Des. 17 (1998) 645-654.

17. G. Chen and E. G. Friedman, Transient simulation of on-chip transmission lines via exact pole extraction, Proc. IEEE Int. Symp. Circuits and Systems, May 2008 (Accepted).

18. M. Kamon, M. J. Tsuk and J. White, FastHenry: A multipole accelerated 3-D inductance extraction program, IEEE Trans. Microwave Theor. Tech. 42 (1994) 1750-1758.

19. K. Nabors and J. White, FastCap: A multipole accelerated 3-D capacitance extraction program, IEEE Trans. Comput.-Aided Des. 10 (1991) 1447-1459.

20. J. Qian, S. Pullela and L. Pillage, Modeling the effective capacitance for the $R C$ interconnect of CMOS gates, IEEE Trans. Comput.-Aided Des. 13 (1994) 1526-1535.

21. K. Agarwal, D. Sylvester and D. Blaauw, An effective capacitance based driver output model for on-chip RLC interconnects, Proc. IEEE/ACM Design Automation Conf., June 2003, pp. 376-381.

22. L. K. Vakati and J. Wang, A new multi-ramp driver model with $R L C$ interconnect load, Proc. IEEE Int. Symp. Circuits and Systems, May 2004, pp. V.269-V.271.

23. Open Source ECSM Format Specification Version 1.2., http://www.cadence.com/ webforms/ecsm. 
24. Composite Current Source Modeling, http://www.synopsys.com/products/solutions/ galaxy/ccs/cc_source.html.

25. J. F. Croix and D. F. Wong, Blade and Razor: Cell and interconnect delay analysis using current-based models, Proc. IEEE/ACM Design Automation Conf., June 2003, pp. 386-389.

26. Y. I. Ismail and E. G. Friedman, Effects of inductance on the propagation delay and repeater insertion in VLSI circuits, IEEE Trans. VLSI Syst. 8 (2000) 195-206.

27. F. Dartu, N. Menezes and L. T. Pileggi, Performance computation for precharacterized CMOS gates with RC loads, IEEE Trans. Comput.-Aided Des. 15 (1996) 544-553.

28. Y. I. Ismail, E. G. Friedman and J. L. Neves, Figures of merit to characterize the importance of on-chip inductance, IEEE Trans. VLSI Syst. 7 (1999) 442-449.

29. B. Krauter and S. Mehrotra, Layout based frequency dependent inductance and resistance extraction for on-chip interconnect timing analysis, Proc. IEEE/ACM Design Automation Conf., June 1998, pp. 303-308.

30. S. Sim, K. Lee and C. Y. Yang, High-frequency on-chip inductance model, IEEE Electron. Dev. Lett. 23 (2002) 740-742.

31. Y. Cao et al., Impact of on-chip interconnect frequency-dependent $R(f) L(f)$ on digital and RF design, IEEE Trans. VLSI Syst. 13 (2005) 158-162. 